\title{
Intraperitoneal injections as an alternative method for micro-CT contrast enhanced detection of murine liver tumors
}

\author{
Nathan Sweeney', Stephen Marchant ${ }^{2}$ \& Jesse D Martinez ${ }^{\star, 3,4}$
}

\section{ABSTRACT}

Micro-computed tomography (micro-CT) coupled with tissue, or vascular, specific contrast agent has emerged as a powerful tool for detecting and monitoring tumor growth in the liver of murine animals. Intravenous injections of contrast agents can be technically challenging and lead to errors that can considerably influence the outcome of a preclinical study, prompting an alternative method. Here we assessed the effectiveness of intraperitoneal injections of polyiodinated triglycerides emulsions (Fenestra LC) in micro-CT imaging of young SCID (8 weeks) and old BALB/c (48 weeks) mice with xenograft or carcinogen-induced liver tumors, respectively, and determined an optimal acquisition time. Utilizing an intraperitoneal injection is a viable alternative administration route for using Fenestra in detection and quantification of murine liver tumor burden.

\section{METHOD SUMMARY}

We report that intraperitoneal injections of polyiodinated triglycerides emulsions are a suitable alternative to the intravenous administration route in detecting and quantifying murine liver tumor burden.

\section{KEYWORDS:}

Fenestra LC • intraperitoneal • intravenous $\cdot$ liver cancer $\cdot$ longitudinal $\cdot$ mice - microcT

'Cancer Biology Graduate Interdisciplinary Program, The University of Arizona, AZ 85724, USA; ${ }^{2}$ Medilumine Inc., Montreal, Quebec H2S $2 X 3$, Canada; ${ }^{3} \mathrm{Cell} \&$ Molecular Medicine, The University of Arizona, AZ 85724, USA; ${ }^{4}$ University of Arizona Cancer Center, The University of Arizona, AZ 85724, USA; *Author for correspondence:jmartinez@uacc.arizona.edu

BioTechniques 66: 214-217 (May 2019) 10.2144/ btn-2018-0162
Contrast-enhanced micro-computed tomography (micro-CT) provides a promising approach for noninvasively studying models of human disease in vivo in small animals. Fenestra LC polyiodinated triglyceride emulsion is a contrast agent that has been used for micro-CT liver tumor imaging in mice and rats [1-3]. It is thought that the lipid spheres of Fenestra LC mimic chylomicron remnants and incorporate apolipoprotein E (APO-E) from plasma for selective targeting of hepatocytes, but not liver tumor cells since liver tumor cells do not contain the APO-E receptor [4,5]. Furthermore, data from Suckow et al. indicate that liver macrophage uptake of Fenestra LC is activated once hepatocyte uptake is saturated [6].

The typical administration route for Fenestra LC is with a tail vein intravenous (IV) injection. IV injections via the tail vein are technically challenging, involve failed attempts, and have been found to involve inter-technologist variability [7-9]. To circumvent these issues, we set out to test the efficacy of intraperitoneal (IP) injections as an alternative administration route for Fenestra LC in a murine tumor model. Previous investigators have reported success with a dual IV and IP injection method of Fenestra, but IP injections of Fenestra LC alone have never been tested to our knowledge $[10,11]$.

In this paper, IP injections of Fenestra LC allowed for identification and quantification of murine liver tumors using micro-CT, and therefore they enable the same applications as with IV injections of Fenestra.

\section{MATERIALS \& METHODS}

\section{Carcinogenic tumors}

Fifteen $(n=15)$ in-house male BALB/c mice (Jackson Laboratory, CA, USA, \#00651) averaging $15.2 \mathrm{~g}$ received an IP injection of diethylnitrosamine (DEN; Sigma, \#N0756) at $25 \mu \mathrm{g} / \mathrm{g}$ of body weight in saline at 15 days of age [12]. The mice were then administered $0.05 \%$ phenobarbital (Sigma, \#P1636) through their drinking water at 21 days of age, which continued until they were sacrificed. Mice were housed in an IACUC compliant facility (under protocol \#14-431) with a 12-h day/night light cycle and had access to standard mouse chow and water ad libitum. All applicable institutional and national guidelines for the care and use of animals were followed.

\section{Xenograft tumors}

Hep3B Cells were acquired from ATCC (VA, USA) and cultured in MEM-10\% fetal bovine serum (NY,USA). The cells were maintained in an incubator at $37^{\circ} \mathrm{C}$ and $5 \% \mathrm{CO}_{2}$ humidified air. Four $(n=4) 5-6$ weeks of age male SCID mice (Taconic, NY, USA, \#CB17SC-M homozygous) were anesthetized with Isoflurane (Western Medical, CA, USA) and inoculated with $10 \times 10^{6}$ Hep3B cells in saline by performing a laparotomy injection in the splenic blood vessels at 9 weeks of age [13]. Mice were housed in an IACUCcompliant facility (under protocol \#07-029) as mentioned above but were given standard drinking water.

\section{Micro-CT set-up}

Mice were imaged using a Siemens Inveon micro-CT scanner (PA, USA), which is a variable zoom cone-beam $x$-ray CT system with the capacity to generate images with a spatial resolution of 20 microns over an $8.4 \mathrm{~cm} \times 5.5 \mathrm{~cm}$ field of view. Images were acquired at $55 \mathrm{kVp}$ with an anode current of 500 and a shutter speed of $500 \mathrm{~ms} /$ frame. Scans completed a full $360^{\circ}$ rotation of the x-ray tube with 450 projections. Reconstructions were generated using the Feldkamp cone-beam algorithm. The axial field of view was set at $55.69 \mathrm{~mm}$ with an effective pixel size of $36.26 \mu \mathrm{m}$ resulting 


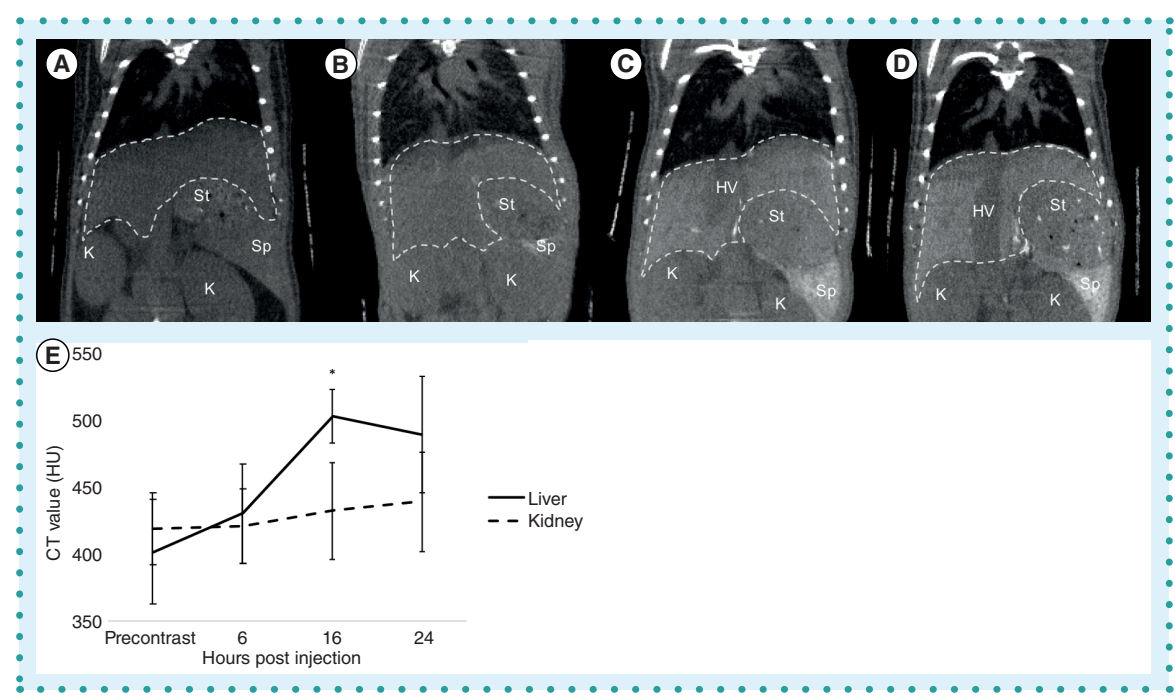

Figure 1. Serial coronal micro-CT images showing hepatobiliary contrast enhancement.

(A) Baseline micro-CT image prior to the contrast agent. A $10 \mathrm{ml} / \mathrm{kg}$ intraperitoneal injection of contrast agent Fenestra LC was injected and micro-CT images were taken at (B) $6 \mathrm{~h},(\mathrm{C}) 16 \mathrm{~h}$, and

(D) $24 \mathrm{~h}$ post-injection. (E) The mean CT value of the liver and kidney at each time point. Error bars represent standard deviation.

*Significant difference in value between liver and kidney ( $p$-value $<0.05$ ).

White dashed line: Liver, St: Stomach, K: Kidney, Sp: Spleen, HV: Hepatic vein.

$\checkmark$ in a reconstruction image size of $2048 \times 3072$ pixels. Final reconstructed data were analyzed using Inveon Research Workplace software.

\section{Administration of contrast agent \& micro-CT imaging}

All mice were manually restrained with one hand and received a $0.5 \mathrm{ml}$ sterile subcutaneous saline injection $24 \mathrm{~h}$ prior to imaging, followed by $10 \mathrm{ml} / \mathrm{kg}$ of the contrast agent, Fenestra $^{\circledR}$ LC (MediLumine Inc., Montreal, Canada) $16 \mathrm{~h}$ prior to imaging for IP injections or $3 \mathrm{ml} / \mathrm{kg}$ of Fenestra LC $2 \mathrm{~h}$ prior to imaging for IV injections, both utilizing a 27-gauge needle. Saline was given per manufacturer recommendation for better contrast agent tolerability. Manually restrained IP injections were injected in the lower right abdominal quadrant of the mice. The syringe plunger was withdrawn, and the needle hub was inspected for urine, blood or digesta. Mice were restrained in a plastic holder (Braintree Scientific, MA, USA) for IV (tail vein) injections. All mice were returned to their home cage until they were imaged. At the time of imaging, mice were placed in an induction chamber and anesthetized with $4 \%$ isoflurane in $0.8-1 \mathrm{l} / \mathrm{min}$ of oxygen. The mice were then transferred to a respiratory pillow on the Inveon bed and kept under anesthesia with approximately $2 \%$ isoflurane in oxygen, depending on the rate of respiration of the mouse. Images were acquired without respiratory or cardiac gating. The total scan time took 11 min with an estimated radiation dose of $80 \mathrm{mSv}$ (PEN dosimeter, S.E. International, TN, USA).

\section{Micro-CT image evaluation}

As mentioned above, micro-CT images were analyzed and measured using Inveon Research Workplace software. Image measurements were determined by their average $\mathrm{CT}$ value $(\mathrm{HU})$, which was determined by the mean voxel intensity from representative slices and locations within the liver, kidney or tumor. Images were blindly analyzed by four trained technicians, whose experience ranged from 4 to 25 years, to determine the reliability of the hepatobiliary segmentation process. The mean CT value and standard deviation were collected for every region of interest and compared between all the images.

\section{Histology}

DEN-exposed mice were euthanized with $\mathrm{CO}_{2}$ gas at 48 weeks post-initiation of treatment exposure. Mice with xenografts were euthanized with $\mathrm{CO}_{2}$ gas at 60 days post cell injection. The livers were harvested, washed in sterile saline, and photographed, then placed in $10 \%$ formalin for $24 \mathrm{~h}$. Once fixed, the livers were transferred to $70 \% \mathrm{EtOH}$ and stored at $4^{\circ} \mathrm{C}$ or embedded in paraffin. The whole livers from all animals were sectioned at $4 \mu \mathrm{m}$ thickness and stained with hematoxylin and eosin and reviewed by a veterinary pathologist.

\section{Statistics}

Using Microsoft Excel (Microsoft, WA, USA) statistical significance was determined using a T-Test. Data are expressed as mean $\pm S D$, and a $p$-value of $\leq 0.05$ was considered statistically significant.

\section{RESULTS \& DISCUSSION}

Liver \& kidney attenuation in micro-CT images from IP injections

As an initial step towards optimizing IP injections, we injected $10 \mathrm{ml} / \mathrm{kg}$ of Fenestra LC; per manufacturer recommendations, into control BALB/c mice $(n=3)$ and evaluated image enhancement over time (pre-contrast, 6,16 and $24 \mathrm{~h}$ ) using serial micro-CT images (Figure 1). We utilized the kidney as our control tissue because of the ease of identifying it among other tissue surrounding the liver. Therefore, liver and kidney enhancement were measured at each time point, and the attenuation difference was compared. As expected, the baseline image (pre-contrast) showed a lack of contrast within the liver and surrounding soft tissues of the mouse (Figure 1A). At $6 \mathrm{~h}$ postinjection contrast enhancement reached a signal intensity of $28.8 \mathrm{HU}$ over liver baseline values and 1.7 HU over kidney baseline values (Figure 1B). Contrast enhancement reached a value of $101.33 \mathrm{HU}$ over liver baseline values as well as $70.5 \mathrm{HU}$ over kidney baseline values at $16 \mathrm{~h}$ post-injection (Figure 1C) which decreased thereafter (Figure 1D). The attenuation difference between the liver and kidney was significant at the 16-h time point with a p-value $<0.05$ (Figure 1E). There was no significant change in kidney enhancement over time.

\section{Comparison of hepatobiliary contrast enhancement by route}

We next compared the liver to tumor enhancement values for IP versus IV injections. We achieved micro-CT hepatobiliary contrast enhancement 16 h post IP $(n=6)$ injection or $2 \mathrm{~h}$, per manufacturer recom- 
mendation, post intravascular $(n=6)$ injection of the contrast agent into mice that were treated with DEN. Although lesions were visualized in micro-CTacquired images, classification of the lesions was determined by a veterinary pathologist post mortem. Liver and tumor enhancement was measured, and the attenuation difference was compared. The mean liver CT values were $575.25 \pm 44.67$ and $520.58 \pm 9.04 \mathrm{HU}$ for the IP and IV contrast agent injections, respectively. The mean tumor CT values were $428.18 \pm 47.31$ and $426.91 \pm 22.14 \mathrm{HU}$ for the IP and IV contrast agent injections, respectively. Therefore, the liver to tumor attenuation differences were $146.97 \pm 2.64$ and $93.67 \pm 13.10 \mathrm{HU}$ for the IP and IV contrast agent injections, respectively. The mean liver CT values were significant with a p-value of less than 0.05 , while the liver to tumor attenuation differences were significant with a p-value of less than 0.001 (Figure 2).

\section{Histopathology confirmed micro-CT detected lesions}

In order to confirm that the imaging using contrast agent delivered by IP yielded suitable images, we first imaged mice that developed tumors as a consequence of being treated with DEN. Contrast-enhanced micro-CT detected a large lesion within the quadrate lobe of the liver from a mouse from the DEN-induced tumor model group (Figure $3 \mathrm{~A}$ ). The harvest confirmed the large $1 \times 1.5 \mathrm{~cm}$ liver lesion, which was photographed, then prepared for histopathology (Figure 3B). The large lesion was determined to be a hepatocellular adenoma with mild cellular hypertrophy and extensive telangiectasia (Figure 3C).

We used the same procedure to determine whether IP-administered contrast agent could detect xenograft liver tumors produced by injection of Hep3B cells into the liver. Contrast-enhanced micro-CT detected multiple lesions of various sizes throughout the liver (Figure 4A). The harvest confirmed multiple liver lesions that ranged from 0.25 to $2 \mathrm{~cm}$ in diameter, which were photographed and prepared for histopathology (Figure 4B). The lesions were determined to be hepatocellular carcinoma with irregular margins that expanded and effaced normal hepatic architecture in multiple liver lobes (Figure 4C).

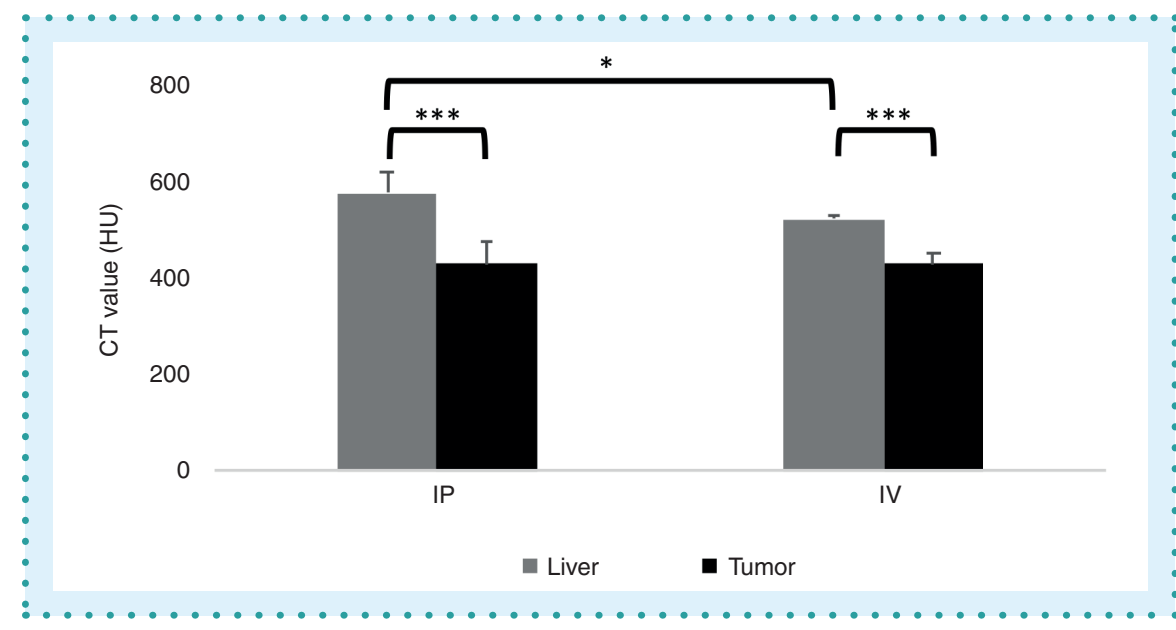

Figure 2. Comparison of contrast enhancement acquired $16 \mathrm{~h}$ post IP $(n=6)$ and $2 \mathrm{~h}$ post IV $(n=6)$ injection. The bar graph represents an average CT value of the liver and tumor tissue. The comparison was done on the same mouse 4 days apart and assessed liver to tumor enhancement as well as the route of injection.

*Significant difference between the route of injection ( $p$-value $<0.05)$.

$* * *$ Significant difference in value between liver and tumor ( $p$-value $<0.001)$.

IP: Intraperitoneal; IV: Intravenous.

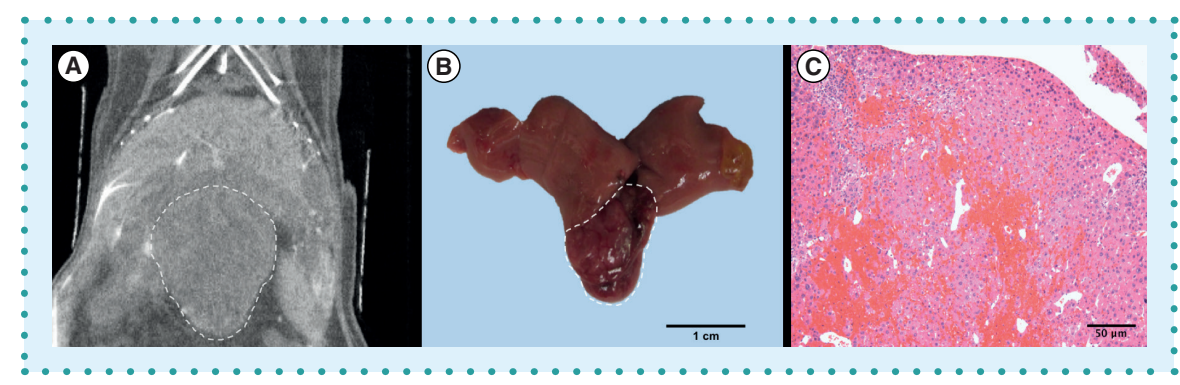

Figure 3. Detection of carcinogen-induced liver tumors using micro-CT. (A) Coronal micro-CT image of a chemically induced tumor, 48 weeks post-DEN injection and phenobarbital administration with large hepatocellular adenoma tumor (dashed line). (B) Upon gross examination of the liver the adenoma was confirmed (dashed line). (C) Hematoxylin and eosin-stained section of the liver with tumor (100x magnification).

This study investigated IP injections of hepatobiliary contrast agent, Fenestra LC, as an alternative to IV injection for detecting and monitoring liver cancer in a murine model. IP injections were suitable for serial imaging as well as imaging mice at multiple time points, which would be ideal for longitudinal drug treatment experiments. Of the time points we tested $(6,16,24 \mathrm{~h})$ our results showed that a 16-h post-intraperitoneal injection image scan provided the best contrast in mice regardless of age or disease burden. These data combined with our observation that the quality of the micro-CT image obtained is comparable to that using IV injections makes IP injection a suitable alternative. In conclusion, our data revealed that IP injections of Fenestra LC produced high-quality micro-CT images of the liver that enabled serial imaging of mice at multiple time points and facilitated the identification of organ tissues within the body and visualization of hepatic adenomas and carcinomas.

\section{FUTURE PERSPECTIVE}

We predict that IP injection as described in this report will be technically easier for investigators to perform, resulting in fewer animal losses while maintaining IV-comparable image quality. As a result, we foresee greater success in longitudinal studies due to the previously mentioned benefits. Future studies comparing Fenestra LC administration by using an oral route could benefit this article, as may studies comparing IP injection of Fenestra LC with other commercially available micro-CT contrast agent. 


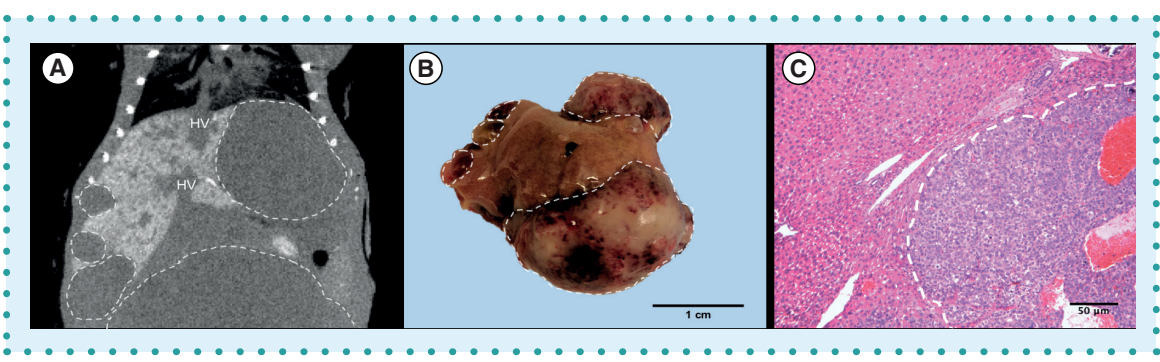

Figure 4. Detection of a xenograft-induced liver tumor using micro-CT. (A) Coronal micro-CT image of a xenograft-induced tumor, 60 days post splenic blood vessel injection of Hep3B cells showing hepatobiliary contrast enhancement and severe hepatocellular carcinoma burden (dashed line). (B) Gross examination confirmed multiple hepatocellular carcinoma tumors (dashed line).

(C) Hematoxylin and eosin-stained section of the liver with tumor (100x magnification).

\section{$\checkmark$ ACKNOWLEDGMENTS}

We would like to give special thanks Bethany Skovan for culturing the Hep3B cells and performing the animal xenografts, Gillian Paine-Murrieta for injecting the contrast agent, Brenda Baggett for micro-CT training, and Veterinary Pathologist David Besselsen, Ph.D, for his interpretation of the histology slides.

\section{AUTHOR CONTRIBUTIONS}

NS championed this experiment which involved: tending the mice, acquiring the micro-CT images, analyzing the images, evaluating the results, and constructing all graphs and figures. SM provided vital expertise. JM helped with interpretation of the data and to draft the manuscript. All authors read and approved the final manuscript.

\section{ETHICAL CONDUCT OF RESEARCH} All applicable Institutional Animal Care and Use Committee (IACUC, \#13-431 and
\#07-029) guidelines for the care and use of animals were followed.

\section{FINANCIAL \& COMPETING INTERESTS DISCLOSURE}

This work was funded by a grant from the National Institute of Health (CA184920) to Jesse D Martinez. The research in this manuscript was directly supported by the Experimental Mouse Shared Resource and the Cancer Imaging Shared Resource at The University of Arizona Cancer Center all of which are funded by the National Cancer Institute Award P30CA023074. The authors have no other relevant affiliations or financial involvement with any organization or entity with a financial interest in or financial conflict with the subject matter or materials discussed in the manuscript apart from those disclosed.

No writing assistance was utilized in the production of this manuscript.

\section{EXECUTIVE SUMMARY}

- Hepatobiliary contrast enhancement was greatest at $16 \mathrm{~h}$ post intraperitoneal (IP) injection.

- Hepatobiliary contrast enhancement was signifi- cantly higher in IP injected mice compared with intravenous (IV) injected mice.

- IP injections detect liver tumors in both carcinogen- and xenograft-induced tumors.

- An IP injection is a comparable alternative for IV injections of Fenestra LC in this liver tumor model.

\section{OPEN ACCESS}

This work is licensed under the AttributionNonCommercial-NoDerivatives 4.0 Unported License. To view a copy of this license, visit http://creativecommons.org/licenses/ by-nc-nd/4.0/

\section{REFERENCES}

1. Bakan DA, Weichert JP, Longino MA, Counsell RE. Polyiodinated triglyceride lipid emulsions for use as hepatoselective contrast agents in CT: effects of physicochemical properties on biodistribution and imaging profiles. Invest. Radiol. 35(3), 158-169 (2000).

2. Ohta S, Lai EW, Morris JC et al. MicroCT for high-resolution imaging of ectopic pheochromocytoma tumors in the liver of nude mice. Int. J. Cancer 119(9), 2236-2241 (2006).

3. Martiniova L, Kotys MS, Thomasson D et al. Noninvasive monitoring of a murine model of metastatic pheochromocytoma: a comparison of contrast-enhanced microCT and nonenhanced MRI. J. Magn. Reson. Imaging 29(3), 685-691 (2009).

4. Hallouard $\mathrm{F}$, Anton $\mathrm{N}$, Choquet $\mathrm{P}$, Constantinesco $\mathrm{A}$, Vandamme T. lodinated blood pool contrast media for preclinical x-ray imaging applications - a review. Biomaterials 31, 6249-6268 (2010).

5. Willekens I, Lahoutte T, Buls $\mathrm{N}$ et al. Time-course of contrast enhancement in spleen and liver with Exia 160, Fenestra LC, and VC. B. Acad. Mol. Imaging Mol. Imaging Biol. 11, 128-135 (2008).

6. Suckow CE, Stout DB. MicroCT liver contrast agent enhancement over time, dose, and mouse strain. Mol. Imaging Biol. 10(2), 114-120 (2008).

7. Groman EV, Reinhardt CP. Method to quantify tail vein injection technique in small animals. Contemp. Top. Lab. Anim. Sci. 43(1), 35-38 (2004)

8. Vines DC, Green DE, Kudo G, Keller H. Evaluation of mouse tail-vein injections both qualitatively and quan titatively on small-animal PET tail scans. J. Nucl. Med. Technol. 39(4), 264-270 (2011).

9. Rampurwala M, Ravoori MK, Wei W, Johnson VE, Vikram R, Kundra V. Visualization and quantification of intraperitoneal tumors by in vivo computed tomography using negative contrast enhancement strategy in a mouse model of ovarian cancer. Transl. Oncol. 2(2), 96-106 (2009).

10. Akladios C, Bour B, Raykov Z, Mutter D, Marescaux J, Aprahamian M. Structural imaging of the pancreas in rat using micro-CT: application to a non-invasive longitudinal evaluation of pancreatic ductal carcinoma monitoring. J. Cancer Res. Ther. 1(2), 70-76 (2013).

11. Ignat $\mathrm{M}$, Akladios $\mathrm{CY}$, Lindner $\mathrm{V}$ et al. Development of a methodology for in vivo follow-up of hepatocellular carcinoma in hepatocyte specific Trim24-null mice treated with myo-inositol trispyrophosphate. J. Exp. Clin. Cancer Res. 35(1), 155 (2016).

12. Klaunig JE, Pereira MA, Ruch RJ, Weghorst CM. Doseresponse relationship of diethylnitrosamine-initiated tumors in neonatal balb/c mice: effect of phenobarbital promotion. Toxicol. Pathol. 16(3), 381-385 (1988).

13. Soares KC, Foley $\mathrm{K}$, Olino $\mathrm{K}$ et al. A preclinical murine model of hepatic metastases. J. Vis. Exp. (91), e51677-e51677 (2014) 